\title{
Transdermal Nitroglycerine Patch: An Optional Device to Reduce Flap Venous Congestion? A Case Report
}

\author{
Sara Di Lorenzo, Bartolo Corradino, Adriana Cordova
}

Department of Surgical Oncology, Plastic Surgery Unit, University of Palermo, Palermo, Italy.

Email: saradilorenz@gmail.com, dilsister@libero.it

Received August $1^{\text {st }}, 2013$; revised September $1^{\text {st }}, 2013$; accepted September $8^{\text {th }}, 2013$

Copyright (C) 2013 Sara Di Lorenzo et al. This is an open access article distributed under the Creative Commons Attribution License, which permits unrestricted use, distribution, and reproduction in any medium, provided the original work is properly cited.

\begin{abstract}
Sometimes in free flap there is a venous congestion without an obstruction of the venous anastomosis or other organic causes of reduction venous drainage (haematoma, seroma compressing the pedicle). In these cases the authors suggest the application of nitroglycerine patch in the congested area of the flap few hours before the surgical exploration of the anastomosis. If there is a fast improvement of the clinical feature of the flap, the surgical exploration could be avoided. The authors underline that applying the nitroglycerin patch should not be regarded in any way as a therapy of a free flap venous thrombosis but only as an useful device, an option to be taken only when the surgeon is undecided whether to revisit the anastomosis or not.
\end{abstract}

Keywords: Free Flap Failure; Venous Congestion; Vascular Pedicle Thrombosis; Microsurgical Complications

\section{Introduction}

The majority of surgical complications after tissue transfer surgery are related to vascular thrombosis, which usually occur within 3 days of surgery, but sometimes thrombosis can occur later.

There are many techniques available for evaluation of flap viability. The most common technique is clinical observation and Doppler vascular pedicle monitoring. The clinical observation is very useful when the flap has a skin island.

Venous thrombosis occurs more frequently because of its low-flow, low-pressure nature, and because it may evolve over several hours.

A venous congestion usually results in oedema and darkening of the skin colour.

During early venous obstruction a needle stick will cause a rapid bleeding of dark blood. The capacity to understand and identify the early signs of flap sufferance is important in order to decide if surgical explorations, and an additional pharmacological therapy or something else are necessary.

If a flap's vascular integrity is in question, if there is a doubt of venous thrombosis at the anastomosis, a prompt surgical exploration is mandatory [1].

Surgical exploration is still mandatory when there are haematoma and seroma that could make a compression on the vascular pedicle.
Sometimes there is a venous congestion of the flap without an obstruction of the venous vessel at the anastomotic site. In the surgical exploration sometimes there is no problem at the anastomotic site or other organic causes of reduction venous drainage.

This kind of insufficient venous drainage may cause a slow partial flap loss that may require further surgical steps, multiple debridements to remove necrotic tissue and prevent further complications as infections, and to improve the outcome.

Authors expose their experience with a nitroglycerine plaster applied on flap in order to reduce the venous congestion of a free DIEP flap. It begun in the 5th day after surgery.

\section{Clinical Case}

The patient is a 38-year-old female undergone a right mastectomy for a ductal carcinoma and a quadrantectomy on the left breast for a dysplasia.

A delayed free Diep Flap reconstruction of the right breast was performed using the internal mammary vessels, as recipient vessels. The ischemia time was less than 2 hours.

The flap seemed well perfused.

The patient didn't smoke and hadn't risk factor or systemic disease.

On 5th day after surgery the patient presented an 
alarming violaceous area in the medial region of the flap, interesting quickly almost half of the flap (Figures 1(a) and (b)). This area corresponded to the area called III of the tram flaps.

The Doppler signal was good and the needle pricked caused the exit of red blood in the lateral area of the flap, black blood in the medial area.

The surgeons told the patient that it could be necessary an exploration of the anastomotic site in operating room, but the patient refused the surgical exploration absolutely.

The authors decided to wait for some hours in order to evaluate the evolution of the clinical deterioration of the flap and in order to exclude the possibility that this cyanotic feature was caused by a temporary problem, as patient position et al.

During this waiting-time a nitroglycerine plaster was applied on the flap, exactly on the violaceous and congested area.

The dosage of the plaster was $10 \mathrm{U}$ nitroglycerine. The limit of the blue area was drawn with a pen in order to evaluate better the increment or the reduction of the suffering area. 4 hours after the plaster application the flap became better.

The surgeons decided to wait.

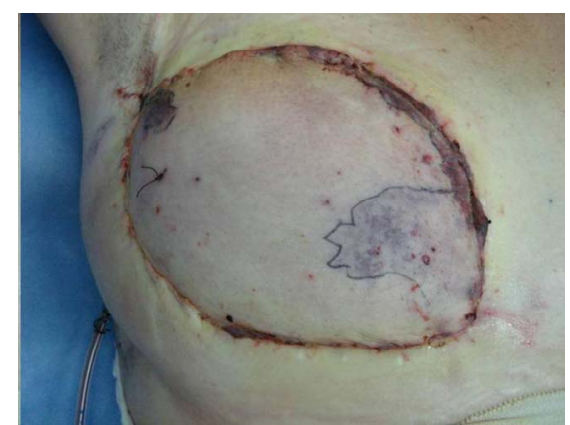

(a)

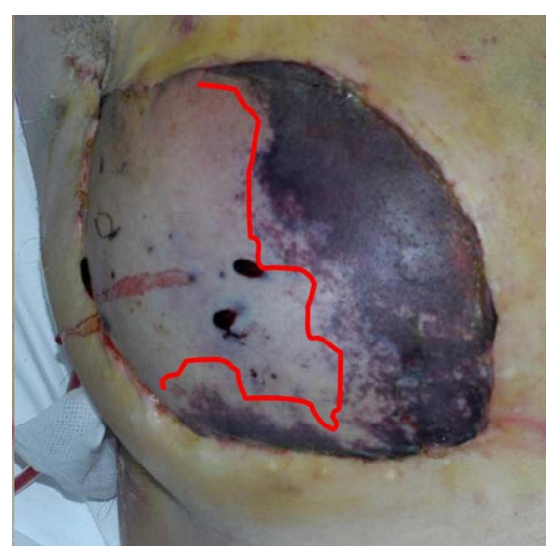

(b)

Figure 1. (a) Violaceous area presented on Diep Flap in 5th day after surgery; (b) Alarming violaceous area developed in few hours, in 5 th day post-operative.
The day after, the flap was better than the day before, it appeared less violaceous and less oedematous.

The patient denied any side effect, onetime headache. The blood pressure was low (105/70) (Figure 2).

The limit line drawn showed a reduction of $1 \mathrm{~cm} /$ die of the violaceous area, that continued to reduce itself in the following days. The plaster was applied every day for 7 days, until the flap became almost all pink (Figures 3-5).

After 2 weeks there weren't signs of congestion and the flap appeared well, with good colour, texture and consistence. A small necrotic area $(2 \mathrm{~cm} \times 2 \mathrm{~cm})$ remained and was surgically removed. The outcome was good (Figure 6).

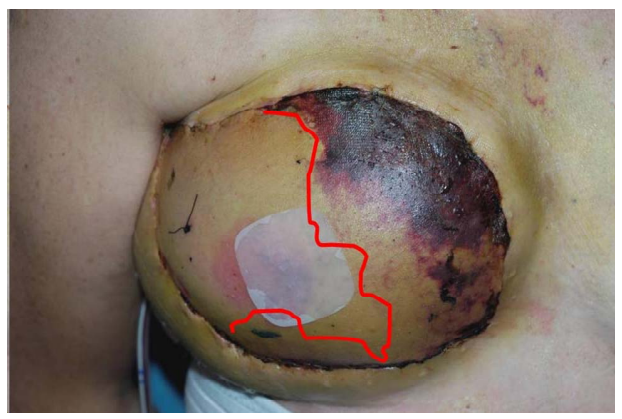

Figure 2. The flap 24 hours after the application of nitroglycerine plaster.

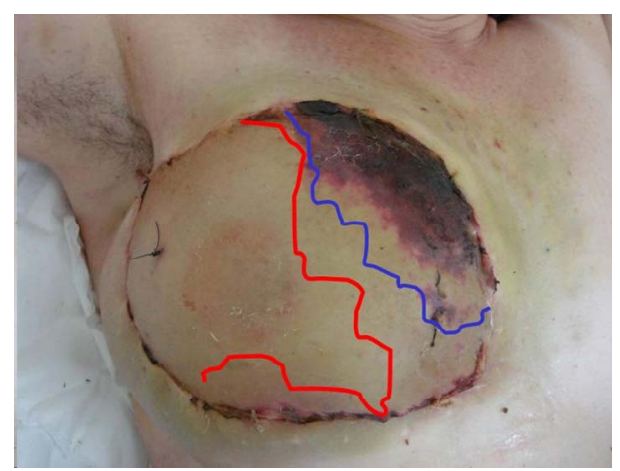

Figure 3. The reduction of the suffering area 4 days after the application of the plaster.

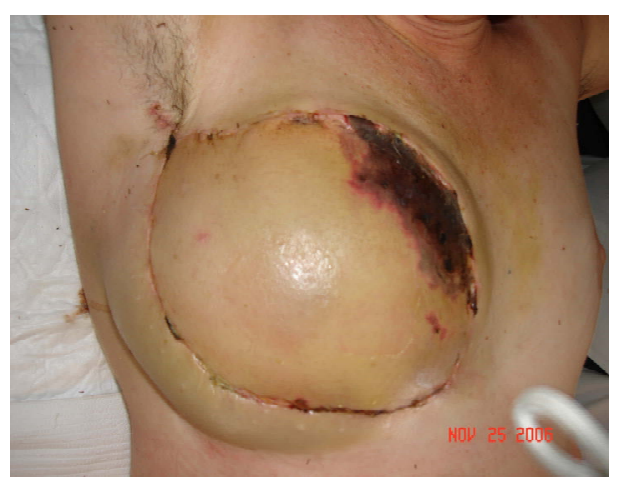

Figure 4. Reduction of the suffering area 5 days after the application of the plaster. 


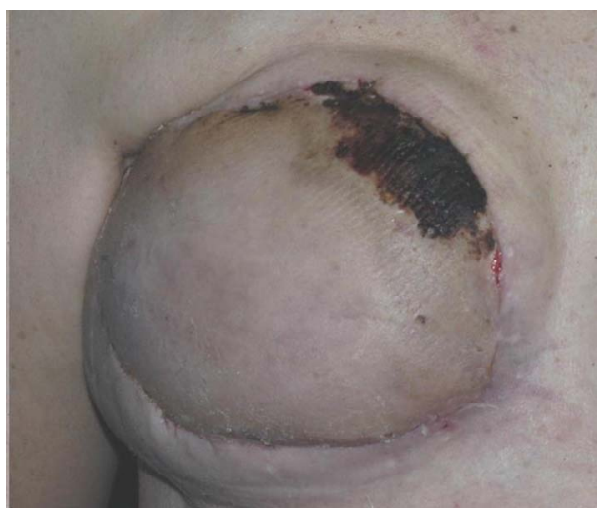

Figure 5. The reduction of the suffering area 6 days after the application of the plaster.

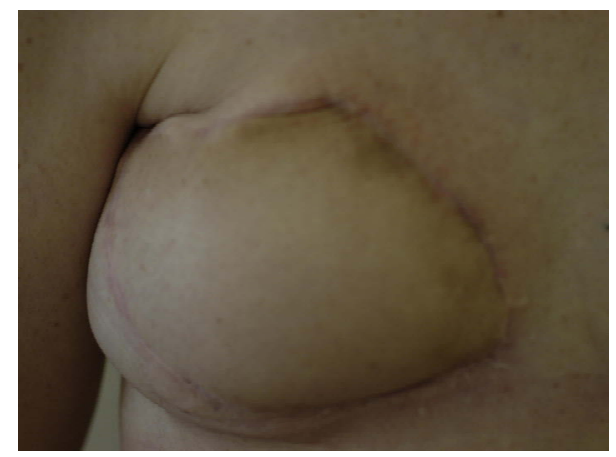

Figure 6. The outcome after 2 weeks.

\section{Conclusion}

Despite the variety of anticoagulation protocols, the studies about the prophylactic anticoagulation therapy and the almost perfect reconstructive techniques used by microsurgeons, the reported failure rate among free flap ranges from $4 \%$ to $10 \%$. The free flap suffering or failure is a complication that often requires, after a rapid recognition of the clinical aspect of the flap, the surgical re-exploration in order to remove haematomas, seroma over the pedicle or to perform a new anastomoses [2]. Sometime the venous congestion of the flap is caused by a temporary problem (spasm) that could not require a surgical revision of the anastomosis. The authors expose their experience with a nitroglycerine plaster applied on flap in order to reduce the venous congestion of a free DIEP flap. It begun in the 5th day after surgery. The use of Nitro-derived drugs in the treatment of angina pectoris is well known. The nitrates caused a dilatation of the venous compartment and then a dilatation of arterial compartment included coronary arteries. Organic nitrates as nitroglycerine, are the most employed drugs in cardiological clinical practice. Nitroglycerine is largely given as a plaster whit transdermal absorption. The mechanism of action of organic nitrates is well known. They act on the endothelial layer inducing the production of NO (nitric oxide). This gas, physiologically produced by endothelial cells, white blood cells, muscular cells and brain cells, caused vasodilatation and reduced the platelet aggregation. NO releasing of endothelial cells (induced by nitroglycerine) causes an increment of level of GMPc inside the smooth muscular cells of the vessels, so the muscular smooth cell relaxation finally causes the vasodilatation of the vessels. The authors expose their experience; it's suggested that during the period, the surgeon is still evaluating if a reexploration of the flap or of the anastomotic site is necessary, and applying a nytroglicerin patch on the suffering flap without local or systemic side effects is possible. If there is a fast improvement of the clinical feature of the flap, the surgical exploration could be avoided.

\section{REFERENCES}

[1] B. C. D. Evans and G. R. D. Evans, "Microvascular Surgery,” Plastic \& Reconstructive Surgery, Vol. 119, No. 2, 2007, pp. 18e-30e. http://dx.doi.org/10.1097/01.prs.0000246717.81077.e4

[2] M. Askari, C. Fisher, F. G. Weniger, S. Bidic and A. W. P. Lee, "Anticoagulation Therapy in Microsurgery: A Review,” Journal of Hand Surgery, Vol. 31, No. 5, 2006, pp. 836-846. 